$\mathrm{J}$ o u r n a l of

Mathematics

and Applications

No 35, pp 69-81 (2012)

\title{
Multiplier sequence spaces of fuzzy numbers defined by a Musielak-Orlicz function
}

\author{
Kuldip Raj and Amit Gupta
}

\begin{abstract}
Submitted by: Marian Mattoka
ABSTRACT: In this paper we introduce some multiplier sequence spaces of fuzzy numbers by using a Musielak-Orlicz function $\mathcal{M}=\left(M_{k}\right)$ and multiplier function $u=\left(u_{k}\right)$ and prove some inclusion relations between the resulting sequence spaces.
\end{abstract}

AMS Subject Classification: 40A05, 40D25

Key Words and Phrases: fuzzy numbers, Musielak-Orlicz function, De La-Vallee Poussin means, Statistical convergence, Multiplier function

\section{Introduction and Preliminaries}

Fuzzy set theory, compared to other mathematical theories, is perhaps the most easily adaptable theory to practice. The main reason is that a fuzzy set has the property of relativity, variability and inexactness in the definition of its elements. Instead of defining an entity in calculus by assuming that its role is exactly known, we can use fuzzy sets to define the same entity by allowing possible deviations and inexactness in its role. This representation suits well the uncertainties encountered in practical life, which make fuzzy sets a valuable mathematical tool. The concepts of fuzzy sets and fuzzy set operations were first introduced by Zadeh [20] and subsequently several authors have discussed various aspects of the theory and applications of fuzzy sets such as fuzzy topological spaces, similarity relations and fuzzy orderings, fuzzy measures of fuzzy events, fuzzy mathematical programming. Matloka [12] introduced bounded and convergent sequences of fuzzy numbers and studied some of their properties. For more details about sequence spaces see ([1], [2], [14], [17]) and refrences therein.

The study of Orlicz sequence spaces was initiated with a certain specific purpose in Banach space theory. Indeed, Lindberg [9] got interested in Orlicz spaces in connection with finding Banach spaces with symmetric Schauder bases having complementary

COPYRIGHT (C) by Publishing Department Rzeszów University of Technology

P.O. Box 85, 35-959 Rzeszów, Poland 
subspaces isomorphic to $c_{0}$. Parashar and Choudhary [16] have introduced and discussed some properties of the sequence spaces defined by using a Orlicz function $M$ which generalized the well-known Orlicz sequence space $l_{M}$ and strongly summable sequence spaces $[\mathcal{C}, 1, p],[\mathcal{C}, 1, p]_{0}$ and $[\mathcal{C}, 1, p]_{\infty}$. Later on, Basarir and Mursaleen [2], Tripathy and Mahanta [19] used the idea of an Orlicz function to construct some spaces of complex sequences. The concept of statistical convergence was introduced by Fast [6] and also independently by Buck [3] and Schoenberg [18] for real and complex sequences. Further this concept was studied by Fridy [7, Connor [4]] and many others. Statistical convergence is closely related to the concept of convergence in Probability.

A fuzzy number is a fuzzy set on the real axis, i.e., a mapping $u: \mathbb{R}^{n} \rightarrow[0,1]$ which satisfies the following four conditions:

1. $u$ is normal, i.e., there exist an $x_{0} \in \mathbb{R}^{n}$ such that $u\left(x_{0}\right)=1$;

2. $u$ is fuzzy convex, i.e., for $x, y \in \mathbb{R}^{n}$ and $0 \leq \lambda \leq 1, u(\lambda x+(1-\lambda) y) \geq$ $\min [u(x), u(y)]$

3. $u$ is upper semi-continuous;

4. the closure of $\left\{x \in \mathbb{R}^{n}: u(x)>0\right\}$, denoted by $[u]^{0}$, is compact.

Denote $L\left(\mathbb{R}^{n}\right)=\left\{u: \mathbb{R}^{n} \rightarrow[0,1] \backslash u\right.$ satisfies (1) - (4) above $\}$. If $u \in L\left(\mathbb{R}^{n}\right)$, then $u$ is called a fuzzy number and $L\left(\mathbb{R}^{n}\right)$ is a fuzzy number space.

Let $C\left(\mathbb{R}^{n}\right)$ denote the family of all non empty, compact, convex subsets of $\mathbb{R}^{n}$. If $\alpha, \beta \in \mathbb{R}$ and $A, B \in C\left(\mathbb{R}^{n}\right)$, then

$$
\alpha(A+B)=\alpha A+\alpha B, \quad(\alpha \beta) A=\alpha(\beta A), \quad 1 A=A
$$

and if $\alpha, \beta \geq 0$, then $(\alpha+\beta) A=\alpha A+\beta A$. The distance between $A$ and $B$ is defined by the Housdorff metric

$$
\delta_{\infty}(A, B)=\max \left\{\sup _{a \in A} \inf _{b \in B}\|a-b\|, \sup _{b \in B} \inf _{a \in A}\|a-b\|\right\},
$$

where $\|$.$\| denoted the usual Euclidean norm in \mathbb{R}^{n}$. It is well known that $\left(C\left(\mathbb{R}^{n}\right), \delta_{\infty}\right)$ is a complete metric space. For $0<\alpha \leq 1$, the $\alpha$-level set $[u]^{\alpha}$ is defined by $[u]^{\alpha}=$ $\left\{x \in \mathbb{R}^{n}: u(x) \geq \alpha\right\}$. Then from (1)-(4), it follows that $[u]^{\alpha} \in\left(C\left(\mathbb{R}^{n}\right)\right)$. For the addition and scalar multiplication in $L\left(\mathbb{R}^{n}\right)$, we have

$$
[u+v]^{\alpha}=[u]^{\alpha}+[v]^{\alpha}, \quad[k u]^{\alpha}=k[u]^{\alpha},
$$

where $u, v \in L\left(\mathbb{R}^{n}\right), k \in \mathbb{R}$. Define, for each $1 \leq q<\infty$,

$$
d_{q}(u, v)=\left(\int_{0}^{1}\left[\delta_{\infty}\left([u]^{\alpha},[v]^{\alpha}\right)\right]^{q}\right)^{\frac{1}{q}}
$$


and $d_{\infty}(u, v)=\sup _{0 \leq \alpha \leq 1} \delta_{\infty}\left([u]^{\alpha},[v]^{\alpha}\right)$, where $\delta_{\infty}$ is the Housdorff metric.

The idea of statistical convergence depends on the density of subsets of the set $\mathbb{N}$ of natural numbers. A subset $E$ of $\mathbb{N}$ is said to have density $\delta(E)$ if

$$
\delta(E)=\lim _{n \rightarrow \infty} \frac{1}{n} \sum_{k=1}^{n} \chi_{E}(k) \text { exists, }
$$

where $\chi_{E}$ is the characteristic function of $E$. It is clear that any finite subset of $\mathbb{N}$ has zero natural density and $\delta\left(E^{c}\right)=1-\delta(E)$.

A sequence $x=\left(x_{k}\right)$ is said to be statistically convergent to the number $L$ if for every $\epsilon>0, \delta\left(\left\{k \in \mathbb{N}:\left|x_{k}-L\right| \geq \epsilon\right\}\right)=0$. In this case, we write $S-\lim x_{k}=L$. A sequence $X=\left(X_{k}\right)$ of fuzzy numbers is said to be bounded if the set $\left\{X_{k}: k \in \mathbb{N}\right\}$ of fuzzy numbers is bounded and convergent to the fuzzy number $X_{0}$, written as $\lim _{k} X_{k}=X_{0}$, i.e if for every $\epsilon>0$ there exists a positive integer $k_{0}$ such that $d\left(X_{k}, X_{0}\right)<\epsilon$, for $k>k_{0}$. By $w^{F}, l_{\infty}^{F}$ and $c^{F}$ denote the set of all, bounded and convergent sequences of fuzzy numbers, respectively see [12].

An Orlicz function is a function $M:[0, \infty) \rightarrow[0, \infty)$ which is continuous, non decreasing and convex with $M(0)=0, M(x)>0$ for $x>0$ and $M(x) \rightarrow \infty$ as $x \rightarrow \infty$. Lindenstrauss and Tzafriri [10] used the idea of Orlicz function to define the following sequence space. Let $w$ be the space of all real or complex sequences $x=\left(x_{k}\right)$, then

$$
l_{M}=\left\{x \in w: \sum_{k=1}^{\infty} M\left(\frac{\left|x_{k}\right|}{\rho}\right)<\infty\right\}
$$

which is called as an Orlicz sequence space. Also $l_{M}$ is a Banach space with the norm

$$
\|x\|=\inf \left\{\rho>0: \sum_{k=1}^{\infty} M\left(\frac{\left|x_{k}\right|}{\rho}\right) \leq 1\right\} .
$$

Also, it was shown in [10] that every Orlicz sequence space $l_{M}$ contains a subspace isomorphic to $l_{p}(p \geq 1)$. The $\Delta_{2}$-condition is equivalent to $M(L x) \leq L M(x)$, for all $L$ with $0<L<1$. An Orlicz function $M$ can always be represented in the following integral form,

$$
M(x)=\int_{0}^{x} \eta(t) d t
$$

where $\eta$ is known as the kernel of $M$, is right differentiable for $t \geq 0, \eta(0)=0, \eta(t)>0$, $\eta$ is non-decreasing and $\eta(t) \rightarrow \infty$ as $t \rightarrow \infty$.

A sequence $\mathcal{M}=\left(M_{k}\right)$ of Orlicz functions is called a Musielak-Orlicz function see ([13],[14]). A sequence $\mathcal{N}=\left(N_{k}\right)$ of Orlicz functions defined by

$$
N_{k}(v)=\sup \left\{|v| u-M_{k}: u \geq 0\right\}, k=1,2, \ldots
$$

is called the complementary function of the Musielak-Orlicz function $\mathcal{M}$. For a given Musielak-Orlicz function $\mathcal{M}$, the Musielak-Orlicz sequence space $t_{\mathcal{M}}$ and its subspace $h_{\mathcal{M}}$ are defined as follows

$$
t_{\mathcal{M}}=\left\{x \in w: I_{\mathcal{M}}(c x)<\infty, \text { for some } c>0\right\}
$$




$$
h_{\mathcal{M}}=\left\{x \in w: I_{\mathcal{M}}(c x)<\infty, \text { for all } c>0\right\},
$$

where $I_{\mathcal{M}}$ is a convex modular defined by

$$
I_{\mathcal{M}}(x)=\sum_{k=1}^{\infty} M_{k}\left(x_{k}\right), x=\left(x_{k}\right) \in t_{\mathcal{M}}
$$

We consider $t_{\mathcal{M}}$ equipped with the Luxemburg norm

$$
\|x\|=\inf \left\{k>0: I_{\mathcal{M}}\left(\frac{x}{k}\right) \leq 1\right\}
$$

or equipped with the Orlicz norm

$$
\|x\|^{0}=\inf \left\{\frac{1}{k}\left(1+I_{\mathcal{M}}(k x)\right): k>0\right\} .
$$

Let $\lambda=\left(\lambda_{n}\right)$ be a non-decreasing sequence of positive numbers tending to $\infty$ and $\lambda_{n+1} \leq \lambda_{n}+1, \lambda_{1}=1$. The generalized De la Vallee-Poussin mean is defined by

$$
t_{n}(x)=\frac{1}{\lambda_{n}} \sum_{k \in I_{n}} x_{k}
$$

where $I_{n}=\left[n-\lambda_{n}+1, n\right]$.

The space $\hat{c}$ of all almost convergent sequences was introduced by Maddox [12] has defined $x=\left(x_{k}\right)$ to be strongly almost convergent to a number $l$ if

$$
\lim _{n} \frac{1}{n} \sum_{k=1}^{n}\left|x_{k+m}-l\right|=0, \text { uniformly in } m .
$$

The following inequality will be used throughout this paper. Let $p=\left(p_{k}\right)$ be a sequence of positive real numbers with $0<p_{k} \leq \sup p_{k}=H$, and let $D=\max \left(1,2^{H-1}\right)$. Then for $a_{k}, b_{k} \in \mathbb{C}$, the set of complex numbers for all $k \in \mathbb{N}$, we have

$$
\left|a_{k}+b_{k}\right|^{p_{k}} \leq D\left\{\left|a_{k}\right|^{p_{k}}+\left|b_{k}\right|^{p_{k}}\right\}
$$

Let $\Lambda$ denote the set of all non-decreasing sequences $\lambda=\left(\lambda_{n}\right)$ of positive numbers tending to $\infty$ such that $\lambda_{n+1} \leq \lambda_{n}+1, \lambda_{1}=1, \mathcal{M}=\left(M_{k}\right)$ be a Musielak-Orlicz function and $p=\left(p_{k}\right)$ be a bounded sequence of positive real numbers. A sequence $X=\left(X_{k}\right)$ of fuzzy numbers is said to be almost $\lambda$-statistically convergent to the fuzzy number $X_{0}$, with respect to the Musielak-Orlicz function, if for every $\epsilon>0$

$$
\lim _{n \rightarrow \infty} \frac{1}{\lambda_{n}}\left|\left\{k \in I_{n}:\left[M_{k}\left(\frac{d\left(t_{k m}(X), X_{0}\right)}{\rho}\right)\right]^{p_{k}} \geq \epsilon\right\}\right|=0,
$$

uniformly in $m$ for some $\rho>0$, 
where the vertical bars indicate the number of elements in the enclosed set and

$$
t_{k m}(X)=\frac{X_{m}+X_{m+1}+\cdots+X_{m+k}}{k+1}=\frac{1}{k+1} \sum_{i=0}^{k} X_{m+i} .
$$

The set of all almost $\lambda$-statistically convergent sequences of fuzzy numbers is denoted by $\hat{S}^{F}\left(\lambda, M_{k}, u, p\right)$. In this case, we write $X_{k} \rightarrow X_{0}\left(\hat{S}^{F}\left(\lambda, M_{k}, u, p\right)\right)$. In the special cases $\lambda_{n}=n$ for all $n \in \mathbb{N}$ and $M_{k}(X)=X, p_{k}=1, u_{k}=1$ for all $k \in \mathbb{N}$, we shall write $\hat{S}^{F}\left(M_{k}, u, p\right)$ and $\hat{S}^{F}(\lambda)$ instead of $\hat{S}^{F}\left(\lambda, M_{k}, u, p\right)$, respectively. Furthermore, the set of all almost statistically convergent sequences of fuzzy numbers is denoted by $\hat{S}^{F}$

Let $\lambda \in \Lambda, \mathcal{M}=\left(M_{k}\right)$ be a Musielak-Orlicz function, $p=\left(p_{k}\right)$ be a bounded sequence of positive real numbers and $u=\left(u_{k}\right)$ be a sequence of strictly positive real numbers. Then we define the following classes of sequences in this paper:

$$
\begin{gathered}
\hat{w}^{F}(\lambda, \mathcal{M}, u, p)=\left\{X=\left(X_{k}\right): \lim _{n \rightarrow \infty} \frac{1}{\lambda_{n}} \sum_{k \in I_{n}} u_{k}\left[M_{k}\left(\frac{d\left(t_{k m}(X), X_{0}\right)}{\rho}\right)\right]^{p_{k}}=0\right. \\
\text { uniformly in } m, \text { for some } \rho>0\} \\
\hat{w}_{0}^{F}(\lambda, \mathcal{M}, u, p)=\left\{X=\left(X_{k}\right): \lim _{n \rightarrow \infty} \frac{1}{\lambda_{n}} \sum_{k \in I_{n}} u_{k}\left[M_{k}\left(\frac{d\left(t_{k m}(X), \overline{0}\right)}{\rho}\right)\right]^{p_{k}}=0\right. \\
\text { uniformly in } m, \text { for some } \rho>0\}
\end{gathered}
$$

and

$$
\begin{aligned}
\hat{w}_{\infty}^{F}(\lambda, \mathcal{M}, u, p)=\{ & X=\left(X_{k}\right): \sup _{m, n} \frac{1}{\lambda_{n}} \sum_{k \in I_{n}} u_{k}\left[M_{k}\left(\frac{d\left(t_{k m}(X), \overline{0}\right)}{\rho}\right)\right]^{p_{k}}<\infty \\
& \text { uniformly in } m, \text { for some } \rho>0\},
\end{aligned}
$$

where

$$
\overline{0}(t)=\left\{\begin{array}{l}
1, \quad t=(0,0,0, \cdots, 0) \\
0, \quad \text { otherwise }
\end{array} .\right.
$$

If $X \in \hat{w}^{F}(\lambda, \mathcal{M}, u, p)$, we say that $X$ is strongly almost $\lambda$-convergent with respect to the Musielak-Orlicz function $\mathcal{M}=\left(M_{k}\right)$. In this case we write $X_{k} \rightarrow$ $X_{0}\left(\hat{w}^{F}(\lambda, \mathcal{M}, u, p)\right)$. The following sequence spaces are defined by giving particular values to $\mathcal{M}, u, p$.

(i) For $\lambda_{n}=n$

$\hat{w}^{F}(\lambda, \mathcal{M}, u, p)=\hat{w}^{F}(\mathcal{M}, u, p), \hat{w}_{0}^{F}(\lambda, \mathcal{M}, u, p)=\hat{w}_{0}^{F}(\mathcal{M}, u, p)$, and $\hat{w}_{\infty}^{F}(\lambda, \mathcal{M}, u, p)=$ $\hat{w}_{\infty}^{F}(\mathcal{M}, u, p)$,

(ii) If $\mathcal{M}=M_{k}(x)=x$ for all $k$, we get 
$\hat{w}^{F}(\lambda, \mathcal{M}, u, p)=\hat{w}^{F}(u, p, \lambda), \hat{w}_{0}^{F}(\lambda, \mathcal{M}, u, p)=\hat{w}_{0}^{F}(u, p, \lambda)$, and $\hat{w}_{\infty}^{F}(\lambda, \mathcal{M}, u, p)=$ $\hat{w}_{\infty}^{F}(u, p, \lambda)$,

(iii) If $p_{k}=1$ for all $k \in \mathbb{N}$, then

$\hat{w}^{F}(\lambda, \mathcal{M}, u, p)=\hat{w}^{F}(\mathcal{M}, u, \lambda), \hat{w}_{0}^{F}(\lambda, \mathcal{M}, u, p)=\hat{w}_{0}^{F}(\mathcal{M}, u, \lambda)$, and $\hat{w}_{\infty}^{F}(\lambda, \mathcal{M}, u, p)=$ $\hat{w}_{\infty}^{F}(\mathcal{M}, u, \lambda)$,

(iv) If $\mathcal{M}=M_{k}(x)=x$ for all $k$, and $p_{k}=1$ for all $k \in \mathbb{N}$, then

$\hat{w}^{F}(\lambda, \mathcal{M}, u, p)=\hat{w}^{F}(u, \lambda), \hat{w}_{0}^{F}(\lambda, \mathcal{M}, u, p)=\hat{w}_{0}^{F}(u, \lambda)$, and $\hat{w}_{\infty}^{F}(\lambda, \mathcal{M}, u, p)=$ $\hat{w}_{\infty}^{F}(u, \lambda)$,

$(v)$ If $p_{k}=1$ for all $k \in \mathbb{N}$, and $u_{k}=1$ for all $k$, then

$\hat{w}^{F}(\lambda, \mathcal{M}, u, p)=\hat{w}^{F}(\mathcal{M}, \lambda), \hat{w}_{0}^{F}(\lambda, \mathcal{M}, u, p)=\hat{w}_{0}^{F}(\mathcal{M}, \lambda)$, and $\hat{w}_{\infty}^{F}(\lambda, \mathcal{M}, u, p)=$ $\hat{w}_{\infty}^{F}(\mathcal{M}, \lambda)$

(vi) If $\mathcal{M}=M_{k}(x)=x, p_{k}=1$ and $u_{k}=1$ for all $k$, then

$\hat{w}^{F}(\lambda, \mathcal{M}, u, p)=\hat{w}^{F}(\lambda), \hat{w}_{0}^{F}(\lambda, \mathcal{M}, u, p)=\hat{w}_{0}^{F}(\lambda)$, and $\hat{w}_{\infty}^{F}(\lambda, \mathcal{M}, u, p)=\hat{w}_{\infty}^{F}(\lambda)$.

In this paper we shall prove properties of linearity and some inclusion relations between the classes of sequences $\hat{w}^{F}(\lambda, \mathcal{M}, u, p), \hat{w}_{0}^{F}(\lambda, \mathcal{M}, u, p), \hat{w}_{\infty}^{F}(\lambda, \mathcal{M}, u, p)$ and $\hat{S}^{F}(\lambda, \mathcal{M}, u, p)$.

\section{Main Results}

Theorem 2.1. For any Musielak-Orlicz function $\mathcal{M}=\left(M_{k}\right), p=\left(p_{k}\right)$ be a bounded sequence of strictly positive real numbers and $u=\left(u_{k}\right)$ be a sequence of positive real numbers, we have

$$
\hat{w}_{0}^{F}(\lambda, \mathcal{M}, u, p) \subset \hat{w}^{F}(\lambda, \mathcal{M}, u, p) \subset \hat{w}_{\infty}^{F}(\lambda, \mathcal{M}, u, p) .
$$

Proof. The inclusion $\hat{w}_{0}^{F}(\lambda, \mathcal{M}, u, p) \subset \hat{w}^{F}(\lambda, \mathcal{M}, u, p)$ is obvious.

Let $X \in \hat{w}^{F}(\lambda, \mathcal{M}, u, p)$, then

$$
\begin{aligned}
\frac{1}{\lambda_{n}} \sum_{k \in I_{n}} u_{k}\left[M_{k}\left(\frac{d\left(t_{k m}(X), \overline{0}\right)}{2 \rho}\right)\right]^{p_{k}} & \leq \frac{D}{\lambda_{n}} \sum_{k \in I_{n}} \frac{1}{2^{p_{k}}} u_{k}\left[M_{k}\left(\frac{d\left(t_{k m}(X), X_{0}\right)}{\rho}\right)\right]^{p_{k}} \\
& +\frac{D}{\lambda_{n}} \sum_{k \in I_{n}} \frac{1}{2^{p_{k}}} u_{k}\left[M_{k}\left(\frac{d\left(X_{0}, \overline{0}\right)}{\rho}\right)\right]^{p_{k}} \\
& \leq \frac{D}{\lambda_{n}} \sum_{k \in I_{n}} u_{k}\left[M_{k}\left(\frac{d\left(t_{k m}(X), X_{0}\right)}{\rho}\right)\right]^{p_{k}} \\
& +D \max _{k \in I_{n}}\left\{\max \left\{1, \sup _{k} u_{k}\left[M_{k}\left(\frac{d\left(X_{0}, \overline{0}\right)}{\rho}\right)\right]^{H}\right\}\right\}
\end{aligned}
$$

where $\sup _{k} p_{k}=H$ and $D=\max \left(1,2^{H-1}\right)$. Thus we get $X \in \hat{w}_{\infty}^{F}(\lambda, \mathcal{M}, u, p)$. 
Theorem 2.2. If $\mathcal{M}=\left(M_{k}\right)$ be a Musielak-Orlicz function, $p=\left(p_{k}\right)$ be a bounded sequence of positive real numbers and $u=\left(u_{k}\right)$ be a sequence of strictly positive real numbers, then $\hat{w}^{F}(\lambda, \mathcal{M}, u, p), \hat{w}_{0}^{F}(\lambda, \mathcal{M}, u, p)$ and $\hat{w}_{\infty}^{F}(\lambda, \mathcal{M}, u, p)$ are closed under the operations of addition and scalar multiplication.

Proof. Let $X=\left(X_{k}\right), Y=\left(Y_{k}\right) \in \hat{w}_{\infty}^{F}(\lambda, \mathcal{M}, u, p)$ and $\alpha, \beta \in \mathbb{C}$. Then there exist positive numbers $\rho_{1}, \rho_{2}$ such that

$$
\sup _{m, n} \frac{1}{\lambda_{n}} \sum_{k \in I_{n}} u_{k}\left[M_{k}\left(\frac{d\left(t_{k m}(X), \overline{0}\right)}{\rho_{1}}\right)\right]^{p_{k}}<\infty, \text { uniformly in } m
$$

and

$$
\sup _{m, n} \frac{1}{\lambda_{n}} \sum_{k \in I_{n}} u_{k}\left[M_{k}\left(\frac{d\left(t_{k m}(Y), \overline{0}\right)}{\rho_{2}}\right)\right]^{p_{k}}<\infty \text {, uniformly in } m .
$$

Define $\rho_{3}=\max \left(2|\alpha| \rho_{1}, 2|\beta| \rho_{2}\right)$. Since $\mathcal{M}=\left(M_{k}\right)$ is non-decreasing and convex, we have

$$
\begin{aligned}
\sup _{m, n} \frac{1}{\lambda_{n}} \sum_{k \in I_{n}} u_{k}\left[M_{k}(\right. & \left.\left.\frac{\alpha d\left(t_{k m}(X), \overline{0}\right)+\beta d\left(t_{k m}(Y), \overline{0}\right)}{\rho_{3}}\right)\right]^{p_{k}} \\
& \leq \frac{1}{2} \sup _{m, n} \frac{1}{\lambda_{n}} \sum_{k \in I_{n}} u_{k}\left[M_{k}\left(\frac{d\left(t_{k m}(X), \overline{0}\right)}{\rho_{1}}\right)\right]^{p_{k}} \\
& +\frac{1}{2} \sup _{m, n} \frac{1}{\lambda_{n}} \sum_{k \in I_{n}} u_{k}\left[M_{k}\left(\frac{d\left(t_{k m}(X), \overline{0}\right)}{\rho_{2}}\right)\right]^{p_{k}} \\
& <\infty .
\end{aligned}
$$

This proves that $\hat{w}_{\infty}^{F}(\lambda, \mathcal{M}, u, p)$ is a linear space. Similarly we can prove for other cases.

Theorem 2.3. If $0<p_{k} \leq r_{k}<\infty$ for all $k \in \mathbb{N}$ and $\left(\frac{r_{k}}{p_{k}}\right)$ be bounded, then we have

$$
\hat{w}_{\infty}^{F}(\lambda, \mathcal{M}, u, r) \subseteq \hat{w}_{\infty}^{F}(\lambda, \mathcal{M}, u, p) .
$$

Proof. Let $X=\left(X_{k}\right) \in \hat{w}_{\infty}^{F}(\lambda, \mathcal{M}, u, r)$. Thus, we have

$$
\sup _{m, n} \frac{1}{\lambda_{n}} \sum_{k \in I_{n}} u_{k}\left[M_{k}\left(\frac{d\left(t_{k m}(X), \overline{0}\right)}{\rho}\right)\right]^{r_{k}}<\infty \text {, uniformly in } m .
$$

Let $s_{k}=\sup _{m, n} \frac{1}{\lambda_{n}} \sum_{k \in I_{n}} u_{k}\left[M_{k}\left(\frac{d\left(t_{k m}(X), \overline{0}\right)}{\rho}\right)\right]^{r_{k}}$ and $\lambda_{k}=\frac{p_{k}}{r_{k}}$. Since $p_{k} \leq r_{k}$, we have $0 \leq \lambda_{k} \leq 1$. Take $0<\lambda<\lambda_{k}$. Now define

$$
u_{k}=\left\{\begin{array}{llr}
s_{k} & \text { if } & s_{k} \geq 1 \\
0 & \text { if } & s_{k}<1
\end{array}\right.
$$


and

$$
v_{k}=\left\{\begin{array}{lll}
0 & \text { if } & s_{k} \geq 1 \\
s_{k} & \text { if } & s_{k}<1
\end{array}\right.
$$

$s_{k}=u_{k}+v_{k}, \quad s_{k}^{\lambda_{k}}=u_{k}^{\lambda_{k}}+v_{k}^{\lambda_{k}}$. It follows that $u_{k}^{\lambda_{k}} \leq u_{k} \leq s_{k}, \quad v_{k}^{\lambda_{k}} \leq v_{k}^{\lambda}$. since $s_{k}^{\lambda_{k}}=u_{k}^{\lambda_{k}}+v_{k}^{\lambda_{k}}$, then $s_{k}^{\lambda_{k}} \leq s_{k}+v_{k}^{\lambda}$

$$
\begin{aligned}
\sup _{m, n} \frac{1}{\lambda_{n}} \sum_{k \in I_{n}} u_{k}\left[\left(M_{k}\left(\frac{d\left(t_{k m}(X), \overline{0}\right)}{\rho_{1}}\right)\right)^{r_{k}}\right]^{\lambda_{k}} & \leq \sup _{m, n} \frac{1}{\lambda_{n}} \sum_{k \in I_{n}} u_{k}\left[M_{k}\left(\frac{d\left(t_{k m}(X), \overline{0}\right)}{\rho_{1}}\right)\right]^{r_{k}} \\
\Longrightarrow \sup _{m, n} \frac{1}{\lambda_{n}} \sum_{k \in I_{n}} u_{k}\left[\left(M_{k}\left(\frac{d\left(t_{k m}(X), \overline{0}\right)}{\rho_{1}}\right)\right)^{r_{k}}\right]^{p_{k} / r_{k}} & \leq \sup _{m, n} \frac{1}{\lambda_{n}} \sum_{k \in I_{n}} u_{k}\left[M_{k}\left(\frac{d\left(t_{k m}(X), \overline{0}\right)}{\rho_{1}}\right)\right]^{r_{k}} \\
\Longrightarrow \sup _{n, m} \frac{1}{\lambda_{n}} \sum_{k \in I_{n}} u_{k}\left[\left(M_{k}\left(\frac{d\left(t_{k m}(X), \overline{0}\right)}{\rho_{1}}\right)\right)\right]^{p_{k}} & \leq \sup _{m, n} \frac{1}{\lambda_{n}} \sum_{k \in I_{n}} u_{k}\left[M_{k}\left(\frac{d\left(t_{k m}(X), \overline{0}\right)}{\rho}\right)\right]^{r_{k}}
\end{aligned}
$$

But

$$
\sup _{m, n} \frac{1}{\lambda_{n}} \sum_{k \in I_{n}} u_{k}\left[M_{k}\left(\frac{d\left(t_{k m}(X), \overline{0}\right)}{\rho}\right)\right]^{r_{k}}<\infty \text {, uniformly in } m \text {. }
$$

Therefore

$$
\sup _{m, n} \frac{1}{\lambda_{n}} \sum_{k \in I_{n}} u_{k}\left[M_{k}\left(\frac{d\left(t_{k m}(X), \overline{0}\right)}{\rho}\right)\right]^{p_{k}}<\infty, \text { uniformly in } m .
$$

Hence $x \in \hat{w}_{\infty}^{F}(\lambda, \mathcal{M}, u, p)$. Thus we get $\hat{w}_{\infty}^{F}(\lambda, \mathcal{M}, u, r) \subseteq \hat{w}_{\infty}^{F}(\lambda, \mathcal{M}, u, p)$.

Theorem 2.4. Suppose $\mathcal{M}=\left(M_{k}\right)$ be a Musielak-Orlicz function, $p=\left(p_{k}\right)$ be a bounded sequence of positive real numbers and $u=\left(u_{k}\right)$ be a sequence of strictly positive real numbers. If $\sup _{k}\left(M_{k}(t)\right)^{p_{k}}<\infty$ for all fixed $t>0$, then

$$
\hat{w}^{F}(\lambda, \mathcal{M}, u, p) \subset \hat{w}_{\infty}^{F}(\lambda, \mathcal{M}, u, p)
$$

Proof. Let $X \in \hat{w}^{F}(\lambda, \mathcal{M}, u, p)$, then there exists a positive number $\rho_{1}>0$ such that

$$
\lim _{n \rightarrow \infty} \frac{1}{\lambda_{n}} \sum_{k \in I_{n}} u_{k}\left[M_{k}\left(\frac{d\left(t_{k m}(X), X_{0}\right)}{\rho_{1}}\right)\right]^{p_{k}}=0 \text {, uniformly in } m .
$$

Define $\rho=2 \rho_{1}$. Since $\mathcal{M}=\left(M_{k}\right)$ is non-decreasing and convex, for each $k$. So by using (1), we have 


$$
\begin{aligned}
\sup _{m, n} \frac{1}{\lambda_{n}} \sum_{k \in I_{n}} u_{k}[ & \left.M_{k}\left(\frac{d\left(t_{k m}(X), \overline{0}\right)}{\rho}\right)\right]^{p_{k}} \\
& \leq \sup _{m, n} \frac{1}{\lambda_{n}} \sum_{k \in I_{n}} u_{k}\left[M_{k}\left(\frac{d\left(t_{k m}(X), X_{0}\right)+d\left(X_{0}, \overline{0}\right)}{\rho}\right)\right]^{p_{k}} \\
& \leq D\left\{\sup _{m, n} \frac{1}{\lambda_{n}} \sum_{k \in I_{n}} \frac{1}{2^{p_{k}}} u_{k}\left[M_{k}\left(\frac{d\left(t_{k m}(X), X_{0}\right)}{\rho_{1}}\right)\right]^{p_{k}}\right. \\
& \left.+\sup _{m, n} \frac{1}{\lambda_{n}} \sum_{k \in I_{n}} \frac{1}{2^{p_{k}}} u_{k}\left[M_{k}\left(\frac{d\left(t_{k m}(X), \overline{0}\right)}{\rho_{1}}\right)\right]^{p_{k}}\right\} \\
& <\infty .
\end{aligned}
$$

Thus $X \in \hat{w}_{\infty}^{F}(\lambda, \mathcal{M}, u, p)$, which completes the proof.

Theorem 2.5. Let $0<h=\inf p_{k} \leq p_{k} \leq \sup p_{k}=H<\infty$. Then for a MusielakOrlicz function $\mathcal{M}=\left(M_{k}\right)$ which satisfies the $\Delta_{2}$-condition, we have $\hat{w}_{0}^{F}(\lambda, u, p) \subset$ $\hat{w}_{0}^{F}(\lambda, \mathcal{M}, u, p), \hat{w}^{F}(\lambda, u, p) \subset \hat{w}^{F}(\lambda, \mathcal{M}, u, p)$ and $\hat{w}_{\infty}^{F}(\lambda, u, p) \subset \hat{w}_{\infty}^{F}(\lambda, \mathcal{M}, u, p)$.

Proof. Let $X \in \hat{w}^{F}(\lambda, u, p)$, then we have

$$
\frac{1}{\lambda_{n}} \sum_{k \in I_{n}} u_{k}\left[\left(\frac{d\left(t_{k m}(X), X_{0}\right)}{\rho}\right)\right]^{p_{k}} \rightarrow 0 \text { as } n \rightarrow \infty \text {, uniformly in } m \text {. }
$$

Let $\epsilon>0$ and choose $\delta$ with $0<\delta<1$ such that $M_{k}(t)<\epsilon$ for $0 \leq t \leq \delta$. Then

$$
\begin{aligned}
\frac{1}{\lambda_{n}} \sum_{k \in I_{n}} u_{k}\left[M_{k}\left(\frac{d\left(t_{k m}(X), X_{0}\right)}{\rho}\right)\right]^{p_{k}} & =\frac{1}{\lambda_{n}} \sum_{k \in I_{n}, d\left(t_{k m}(X), X_{0}\right) \leq \delta} u_{k}\left[M_{k}\left(\frac{d\left(t_{k m}(X), X_{0}\right)}{\rho}\right)\right]^{p_{k}} \\
& +\frac{1}{\lambda_{n}} \sum_{k \in I_{n}, d\left(t_{k m}(X), X_{0}\right)>\delta} u_{k}\left[M_{k}\left(\frac{d\left(t_{k m}(X), X_{0}\right)}{\rho}\right)\right]^{p_{k}} \\
& =\sum_{1}+\sum_{2} .
\end{aligned}
$$

where

$$
\sum_{1}=\frac{1}{\lambda_{n}} \sum_{k \in I_{n}, d\left(t_{k m}(X), X_{0}\right) \leq \delta} u_{k}\left[M_{k}\left(\frac{d\left(t_{k m}(X), X_{0}\right)}{\rho}\right)\right]^{p_{k}}<\max \left(\epsilon, \epsilon^{H}\right)
$$

by using continuity of $\left(M_{k}\right)$. For the second summation, we will make the following procedure. Thus we have

$$
\frac{d\left(t_{k m}(X), X_{0}\right)}{\rho}<1+\frac{d\left(t_{k m}(X), X_{0}\right) / \rho}{\delta}
$$


Since $\mathcal{M}=\left(M_{k}\right)$ is non-decreasing and convex, so we have

$$
\begin{aligned}
u_{k}\left[M_{k}\left(\frac{d\left(t_{k m}(X), X_{0}\right)}{\rho}\right)\right] & <u_{k}\left[M_{k}\left\{1+\frac{d\left(t_{k m}(X), X_{0}\right) / \rho}{\delta}\right\}\right] \\
& \leq \frac{1}{2} u_{k}\left[M_{k}(2)\right]+\frac{1}{2} u_{k}\left[M_{k}\left\{2 \frac{d\left(t_{k m}(X), X_{0}\right) / \rho}{\delta}\right\}\right] .
\end{aligned}
$$

Again, since $\mathcal{M}=\left(M_{k}\right)$ satisfies the $\Delta_{2}$-condition, it follows that

$$
\begin{aligned}
u_{k}\left[M_{k}\left(\frac{d\left(t_{k m}(X), X_{0}\right)}{\rho}\right)\right] & \leq \frac{1}{2} L\left\{\frac{d\left(t_{k m}(X), X_{0}\right) / \rho}{\delta}\right\} u_{k}\left[M_{k}(2)\right] \\
& +\frac{1}{2} L\left\{\frac{d\left(t_{k m}(X), X_{0}\right) / \rho}{\delta}\right\} u_{k}\left[M_{k}(2)\right] \\
& =L\left\{\frac{d\left(t_{k m}(X), X_{0}\right) / \rho}{\delta}\right\} u_{k}\left[M_{k}(2)\right]
\end{aligned}
$$

Thus, it follows that

$$
\sum_{2}=\max _{k \in I_{n}}\left\{1,\left[\frac{L u_{k}\left[M_{k}(2)\right]}{\delta}\right]^{H}\right\} \frac{1}{\lambda_{n}} \sum_{k \in I_{n}}\left[\left(\frac{d\left(t_{k m}(X), X_{0}\right)}{\rho}\right)\right]^{p_{k}} .
$$

Taking the limit as $\epsilon \rightarrow 0$ and $n \rightarrow \infty$, it follows that $X \in \hat{w}^{F}(\lambda, \mathcal{M}, u, p)$. Similarly, we can prove that $\hat{w}_{0}^{F}(\lambda, u, p) \subset \hat{w}_{0}^{F}(\lambda, \mathcal{M}, u, p)$ and $\hat{w}_{\infty}^{F}(\lambda, u, p) \subset \hat{w}_{\infty}^{F}(\lambda, \mathcal{M}, u, p)$.

Theorem 2.6. If $\mathcal{M}=\left(M_{k}\right)$ be a Musielak-Orlicz function, $p=\left(p_{k}\right)$ be a bounded sequence of positive real numbers and $u=\left(u_{k}\right)$ be a sequence of strictly positive real numbers, then

(i) If $0<\inf p_{k} \leq p_{k} \leq 1$ for all $k$, then $\hat{w}^{F}(\lambda, \mathcal{M}, u) \subseteq \hat{w}^{F}(\lambda, \mathcal{M}, u, p)$,

(ii) If $1 \leq p_{k} \leq \sup p_{k}=H<\infty$ then $\hat{w}^{F}(\lambda, \mathcal{M}, u, p) \subseteq \hat{w}^{F}(\lambda, \mathcal{M}, u)$.

Proof. $(i)$ Let $X \in \hat{w}^{F}(\lambda, \mathcal{M}, u)$. Since $0<\inf p_{k} \leq p_{k} \leq 1$, we get

$$
\frac{1}{\lambda_{n}} \sum_{k \in I_{n}} u_{k}\left[M_{k}\left(\frac{d\left(t_{k m}(X), X_{0}\right)}{\rho}\right)\right]^{p_{k}} \leq \frac{1}{\lambda_{n}} \sum_{k \in I_{n}} u_{k}\left[M_{k}\left(\frac{d\left(t_{k m}(X), X_{0}\right)}{\rho}\right)\right]
$$

and hence $X \in \hat{w}^{F}(\lambda, \mathcal{M}, u, p)$.

(ii) Let $X \in \hat{w}^{F}(\lambda, \mathcal{M}, u, p)$ and $1 \leq p_{k} \leq \sup p_{k}=H<\infty$. Then for every $0<\epsilon<1$, there exists a positive integer $n_{0}$ such that

$$
\frac{1}{\lambda_{n}} \sum_{k \in I_{n}} u_{k}\left[M_{k}\left(\frac{d\left(t_{k m}(X), X_{0}\right)}{\rho}\right)\right]^{p_{k}} \leq \epsilon<1
$$

for all $n \geq n_{0}$. This follows that

$$
\frac{1}{\lambda_{n}} \sum_{k \in I_{n}} u_{k}\left[M_{k}\left(\frac{d\left(t_{k m}(X), X_{0}\right)}{\rho}\right)\right] \leq \frac{1}{\lambda_{n}} \sum_{k \in I_{n}} u_{k}\left[M_{k}\left(\frac{d\left(t_{k m}(X), X_{0}\right)}{\rho}\right)\right]^{p_{k}} .
$$


and hence $X \in \hat{w}^{F}(\lambda, \mathcal{M}, u)$.

Theorem 2.7. If $\mathcal{M}=\left(M_{k}\right)$ be a Musielak-Orlicz function, $p=\left(p_{k}\right)$ be a bounded sequence of positive real numbers, $u=\left(u_{k}\right)$ be a sequence of strictly positive real numbers and $0<h=\inf p_{k} \leq p_{k} \leq \sup p_{k}=H<\infty$. Then $\hat{w}^{F}(\lambda, \mathcal{M}, u, p) \subset \hat{S}^{F}(\lambda)$.

Proof. The proof of the theorem follows from the following inequality:

$$
\begin{aligned}
& \frac{1}{\lambda_{n}} \sum_{k \in I_{n}} u_{k}\left[M_{k}\left(\frac{d\left(t_{k m}(X), X_{0}\right)}{\rho}\right)\right]^{p_{k}} \\
& \geq \frac{1}{\lambda_{n}} \sum_{k \in I_{n}, d\left(t_{k m}(X), X_{0}\right) \geq \epsilon} u_{k}\left[M_{k}\left(\frac{d\left(t_{k m}(X), X_{0}\right)}{\rho}\right)\right]^{p_{k}} \\
& \geq \frac{1}{\lambda_{n}} \sum_{k \in I_{n}, d\left(t_{k m}(X), X_{0}\right) \geq \epsilon} \min \left\{u_{k}\left[M_{k}\left(\epsilon_{1}\right)\right]^{h}, u_{k}\left[M_{k}\left(\epsilon_{1}\right)\right]^{H}\right\} \\
& \geq \frac{1}{\lambda_{n}}\left|\left\{k \in I_{n}: d\left(t_{k m}(X), X_{0}\right) \geq \epsilon\right\}\right| \min _{k \in I_{n}}\left\{u_{k}\left[M_{k}\left(\epsilon_{1}\right)\right]^{h}, u_{k}\left[M_{k}\left(\epsilon_{1}\right)\right]^{H}\right\},
\end{aligned}
$$

where $\epsilon_{1}=\frac{\epsilon}{\rho}$.

Theorem 2.8. Let $\mathcal{M}=\left(M_{k}\right)$ be a Musielak-Orlicz function, $X=\left(X_{k}\right)$ be a bounded sequence of fuzzy numbers and $0<h=\inf p_{k} \leq p_{k} \leq \sup p_{k}=H<\infty$. Then $\hat{S}^{F}(\lambda) \subset \hat{w}^{F}(\lambda, \mathcal{M}, u, p)$.

Proof. Suppose that $X \in l_{\infty}^{F}$ and $X_{k} \rightarrow X_{0}\left(\hat{S}^{F}(\lambda)\right)$. Since $X \in l_{\infty}^{F}$, there exists a constant $K>0$ such that $d\left(t_{k m}(X), X_{0}\right) \leq K$ for all $k, m$. Given $\epsilon>0$, we have

$$
\begin{aligned}
& \frac{1}{\lambda_{n}} \sum_{k \in I_{n}} u_{k}\left[M_{k}\left(\frac{d\left(t_{k m}(X), X_{0}\right)}{\rho}\right)\right]^{p_{k}} \\
& \quad=\frac{1}{\lambda_{n}} \sum_{k \in I_{n}, d\left(t_{k m}(X), X_{0}\right) \geq \epsilon} u_{k}\left[M_{k}\left(\frac{d\left(t_{k m}(X), X_{0}\right)}{\rho}\right)\right]^{p_{k}} \\
& \quad+\frac{1}{\lambda_{n}} \sum_{k \in I_{n}, d\left(t_{k m}(X), X_{0}\right)<\epsilon} u_{k}\left[M_{k}\left(\frac{d\left(t_{k m}(X), X_{0}\right)}{\rho}\right)\right]^{p_{k}} \\
& \quad \leq \frac{1}{\lambda_{n}} \sum_{k \in I_{n}, d\left(t_{k m}(X), X_{0}\right) \geq \epsilon} \max \left\{u_{k}\left[M_{k}\left(\frac{K}{\rho}\right)\right]^{h}, u_{k}\left[M_{k}\left(\frac{K}{\rho}\right)\right]^{H}\right\} \\
& \quad+\frac{1}{\lambda_{n}} \sum_{k \in I_{n}, d\left(t_{k m}(X), X_{0}\right)<\epsilon} u_{k}\left[M_{k}\left(\frac{\epsilon}{\rho}\right)\right]^{p_{k}} \\
& \quad \leq \max _{k \in I_{n}}\left\{u_{k}\left[M_{k}(T)\right]^{h}, u_{k}\left[M_{k}(T)\right]^{H}\right\} \frac{1}{\lambda_{n}}\left|\left\{k \in I_{n}: d\left(t_{k m}(X), X_{0}\right) \geq \epsilon\right\}\right| \\
& \quad+\max _{k \in I_{n}}\left\{u_{k}\left[M_{k}\left(\epsilon_{1}\right)\right]^{h}, u_{k}\left[M_{k}\left(\epsilon_{1}\right)\right]^{H}\right\} .
\end{aligned}
$$

where $T=\frac{K}{\rho}, \frac{\epsilon}{\rho}=\epsilon_{1}$. Hence $X \in \hat{w}^{F}(\lambda, \mathcal{M}, u, p)$. 


\section{References}

[1] M. Basarir, S. Altuntag and M. Kayikci, On some generalized sequence spaces of fuzzy numbers defined by a sequence of Orlicz functions, Rendiconti del Circolo Matematico di Palermo, 59 (2010), 277-287.

[2] M. Basarir and M. Mursaleen,Some sequence spaces of fuzzy numbers generated by infinite matrices, J. fuzzy Math, 11 (2003), 757-764.

[3] R. C. Buck, Generalized Asymptote Density, Amer J. of Math, 75 (1953), 335346.

[4] J. S. Connor, The statistical and strong P-Cesaro convergence of sequences, Analysis, 8 (1988), 47-63.

[5] J. S. Connor, J. A. Fridy and J. Kline, Statistically Pre-Cauchy Sequences, Analysis, 14 (1994), 311-317.

[6] H. Fast, Sur la convergence statistiue, Colloq. Math, 2 (1951), 241-244.

[7] J. A. Fridy, On statistical convergence, Analysis, 5 (1985), 301-313.

[8] M. A. Krasnoselskii and Y. B. Rutickii, Convex functions and Orlicz spaces, Groningen, Netherlands, (1961).

[9] K. Lindberg, On subspace of Orlicz sequence spaces, Studia Math. 45 (1973), 379-390.

[10] J. Lindenstrauss and L. Tzafriri, On Orlicz sequence spaces, Israel J. Math. 10 (1971), 379-390.

[11] I. J. Maddox, A new type of convergence, Math. Proc. Camb. Phil.Soc., 83 (19878), 61-64.

[12] M. Matloka, Sequences of fuzzy numbers, BUSEFAL, 28 (1986), 28-37.

[13] L. Maligranda, Orlicz spaces and interpolation, Seminars in Mathematics 5, Polish Academy of Science, 1989.

[14] J. Musielak, Orlicz spaces and modular spaces, Lecture Notes in Mathematics, 1034 (1983).

[15] S. Nanda, On sequences of fuzzy numbers, fuzzy sets and Systems, 33 (1989), 123-126.

[16] S. D. Parashar and B. Choudhary, Sequence spaces defined by Orlicz functions, Indian J. Pure Appl. Math., 25 (1994), 419-428. 
[17] K. Raj, A.K.Sharma and S. K. Sharma, A sequence space defined by MusielakOrlicz functions, Int. Journal of Pure and Appl. Mathematics, 67 (2011), 475-484.

[18] I. J. Schoenberg, The integrability of certain functions and relatedr summability methods, Amer. Math. Monthly, 66 (1959), 361-375.

[19] B.C. Tripathy and S. Mahanta, On a class of sequences related to the $l_{p}$ spaces defined by Orlicz function, Soochow J. Math., 29 (2003), 379-391.

[20] L. A. Zadeh, Fuzzy sets, Information and control, 8 (1965), 338-353.

DOI: $10.7862 / \mathrm{rf.2012.6}$

\section{Kuldip Raj}

email: kuldipraj68@gmail.com

\section{Amit Gupta}

email: kuldeepraj68@rediffmail.com

School of Mathematics

Shri Mata Vaishno Devi University,

Katra-182320 J \& K, India.

Received 18.08.2011 